\title{
LOS PROCEDIMIENTOS SEMÁNTICOS EN LA FORMACIÓN DE PALABRAS
}

\author{
EUGENIO COSERIU
}

A la memoria de Charles Bally

1.1. En este artículo ${ }^{1}$, nos proponemos presentar de una manera muy sucinta nuestra teoría semántica acerca de la formación de palabras («inhaltliche Wortbildungslehre»), teoría que hemos elaborado en nuestros trabajos sobre semántica estructural (cf. E. Coseriu, Principios de semántica estructural, Madrid, 1977). Dedicamos este esbozo a la memoria de Charles Bally para poner de manifiesto nuestra deuda con él y porque nos parece que el gran maestro ginebrino, aunque tratando otros problemas, identificó en su Linguistique générale et linguistique française $e^{2}$ la mayoría de los procedimientos y de las relaciones semánticas que intervienen en el fenómeno en cuestión.

1.2. Teoría «semántica» significa teoría desarrollada desde el punto de vista del significado e integrada en la semántica estructural y, por lo tanto, en la lingüística funcional, es decir, en la lingüística relativa a las lenguas como sistemas estructurados de expresión y de contenido lingüísticos. En este sentido, el significado es el contenido proporcionado por la lengua, exclusivamente en cuanto sistema de funciones distintivas y opositivas, y debe ser cuidadosamente distinguido de la designación, que, al contrario, es la relación existente entre los signos y la realidad extralingüística nombrada por éstos; es decir, la aplicación de

\footnotetext{
${ }^{1}$ N. de la T.: Este artículo ha sido traducido por Elena Llamas Pombo del original francés: Eugenio Coseriu, «Les procédés sémantiques dans la formation des mots», Cahiers Ferdinand de Saussure, 35, 1981 (Ginebra: Droz, 1982), págs. 3-16. Dicha publicación recoge una conferencia pronunciada por el profesor Coseriu el 21 de mayo de 1981 en la Universidad de Ginebra, intervención que inauguró la serie de Conférences Charles Bally, que anualmente convoca esta Universidad en memoria del lingüista suizo.

El texto en francés es una nueva redacción de la primera versión de este estudio, que fue publicada originariamente en alemán («Inhaltliche Wortbildungslehre (am Beispiel des Typs coupe-papier)», en H. E. Brekle y D. Kastovsky, eds. Perspektiven der Wortbildungsforschung, Bonn, 1977, págs. 48-61) y traducida al español por Marcos Martínez Hernández («La formación de palabras desde el punto de vista del contenido (A propósito del tipo “coupe-papier”)», Gramática, semántica, universales. Estudios de lingüistica funcional, Madrid: Gredos, 1978 [2 $2^{\mathrm{a}}$ ed.: Madrid: Gredos, 1987, págs. 239-264]). Aunque desafortunadamente no llegó a revisar la versión definitiva, el profesor Coseriu dio su aprobación para la publicación de la presente traducción.

${ }^{2}$ N. de la T.: Charles Bally, Linguistique générale et linguistique française, París: Ernest Leroux, 1932.
}

Odisea, $n^{0} 3,2003$, ISSN 1578-3820, pags. 179-189 
los significados a la «realidad». Tal aplicación no está determinada solamente por el sistema lingüístico - que, como tal, es un sistema de virtualidades o posibilidades-, sino también por el conocimiento de las «cosas» y por la norma (realización tradicional o «uso») de la lengua en cuestión. La designación, por consiguiente, puede considerarse como determinación ulterior y complementaria de los procedimientos de formación, pero no puede considerarse antes del significado ni, tampoco, como determinación primaria o definitoria. Ello implica que la teoría semántica, en el sentido que acabamos de precisar, es la única que puede explicar los procedimientos de formación de palabras, como conjunto de procedimientos funcionales de una lengua dada.

2.1. Efectivamente, en su sentido propio, la expresión «formación de palabras» designa - o debería designar- el conjunto de procedimientos más o menos regulares de los que dispone una lengua para formar lexemas secundarios («derivados» y «compuestos») a partir de lexemas que ya posee (y que, por lo demás, pueden ser a su vez lexemas «secundarios»). Ahora bien, las diferentes teorías sobre la formación de palabras -tanto las teorías tradicionales como la mayoría de las teorías modernas- no pueden responder adecuadamente a este fenómeno, en el mismo sentido que intuitivamente reconocen los hablantes y que se manifiesta en la actividad lingüística de éstos. A nuestro parecer, ello es debido a dos razones principales: por una parte, se quiere aludir, simultánea o alternativamente, tanto a la expresión como al contenido; por otra parte, se confunden $-\mathrm{o}$, al menos, no se distinguen constantemente y de modo coherente-designación y significado de lengua.

2.2. Debido a la tendencia a tomar en consideración simultáneamente la expresión y el contenido, sólo se distinguen dos tipos fundamentales de formación de palabras, la derivación y la composición, a pesar de que, por ejemplo, los «derivados» del tipo ruelle o vieillot son completamente diferentes, desde el punto de vista semántico, de otros «derivados» como beauté o sortie, y a pesar de que los derivados del tipo chasseur, vendeur, etc. son más afines, en su estructura semántica, a las palabras compuestas que a los otros «derivados». Por esta misma razón, la formación mediante prefijos (como prévoir o parvenir) se atribuye con bastante frecuencia a la composición, y se habla de «composición verbal» (porque los prefijos corresponden casi siempre a preposiciones que existen como «palabras» autónomas de la lengua), a pesar de que estos «compuestos» no son diferentes, en su estructura semántica, de toda una serie de palabras «derivadas»; y así, naturalmente, se encuentran dificultades en el caso de los prefijos que no existen como palabras autónomas: una formación del tipo revenir, ¿debe ser atribuida a la derivación o a la composición? Por razones análogas, se considera como un tipo particular la formación llamada «parasintética» (por ejemplo, it. sfacciato, fannullone, fruttivendolo, pescivendolo, esp. pordiosero, alem. Dickhäuter, Einhänder, Vierfüßer, dickköpfig), tipo que correspondería simultáneamente a la formación con sufijos y prefijos o a la derivación y a la composición (dado que-según se dice-facciato, nullone, vendolo, diosero, Häuter, Händer, Füßer y köpfig no existen como palabras autónomas). Sin embargo, desde el punto de vista funcional, este tipo es completamente heterogéneo, y no se ve claramente por qué habríamos de partir de diosero, köpfig, etc., y no de por Dios, Dickkopf, etc., que sí existen, admitiendo así formaciones tales como por Dios + -ero y Dickkopf + -ig.

Odisea, $n^{\circ} 3,2003$ 
2.3.1. En cuanto a la no distinción entre designación y significado de lengua, el caso más sintomático es, sin duda, el de los compuestos llamados «endocéntricos»y «exocéntricos». Serían «exocéntricos» Dickkopf, Dummkopf, Rotkäppchen, rouge-gorge y magnanimus, porque lo que designan no es algo que expresan ellos mismos (un «Kopf», un «Käppchen», una «gorge», un «animus»), sino algo exterior a ellos. Al contrario, Haustier, Rotwein y Großstadt serían compuestos «endocéntricos», porque designan algo que constituye al mismo tiempo su determinatum (un «Tier», un «Wein», una «Stadt»). Y, naturalmente, un compuesto como Goldhaar debería ser endocéntrico o exocéntrico, según los casos considerados: sería endocéntrico si designara "cabello dorado» y exocéntrico, si designara una «persona con el cabello dorado». Ahora bien, desde el punto de vista del significado de lengua y de los procedimientos semánticos de formación de palabras, no hay compuestos exocéntricos; sólo hay compuestos endocéntricos: Dickkopf pertenece exactamente al mismo tipo que Rotwein y, en rigor, designa en primer lugar su determinatum «Kopf», del mismo modo que Rotwein designa el determinatum «Wein». El carácter «exocéntrico» no concierne al significado de lengua ni al procedimiento de composición como tal, sino únicamente a la designación por antonomasia. Pero la antonomasia no es un significado, es el empleo de un significado; y es éste un fenómeno que encontramos también en el caso de muchos sintagmas libres e, incluso, de palabras simples (cf., por ejemplo, el empleo antonomástico de böse Zunge, mauvaise langue, chaperon rouge). La única diferencia es que, en el caso de los compuestos llamados exocéntricos, se trata de una designación antonomástica tradicional o fija y, en tal caso, la designación «propia» queda normalmente excluida. Pero esto se encuentra también en el caso de algunas palabras no compuestas (cf., por ejemplo, el empleo fijo de Stift por «Lehrling», «aprendiz», y la designación exclusivamente metafórica de fr. pieds-noirs) e, inversamente, tenemos compuestos «exocéntricos» que pueden emplearse en su sentido propio (como en el caso de Goldhaar). En el fondo, el fenómeno de la exocentricidad no concierne a la composición, sino a la denominación: el hecho de dar tal o cual nombre a tal o cual objeto.

2.3.2. También concierne a la designación la teoría que pretende «explicar» las palabras compuestas -como Papierkorb, Goldwaage, Sommerhut o Filzhut-relacionándolas con oraciones concretas como «Der Korb ist für das Papier», «Die Waage ist für (aus) Gold», «Der Hut ist für den Sommer», «Der Hut ist aus Filz», etc., lo cual rompe la unidad funcional «de lengua» de estos compuestos, ya que, por ejemplo, Goldwaage debería corresponder a dos compuestos diferentes, según se trate de una balanza para oro o de una balanza de oro. Realmente, en lo concerniente al significado de lengua, las relaciones gramaticales dentro de las palabras compuestas son mucho más abstractas y genéricas; así, por ejemplo, en el caso de Papierkorb, tenemos, aproximadamente: «Korb -función preposicionalPapier», es decir, «cesto que tiene algo que ver con el papel». No es más que una relación como tal, una función preposicional genérica, que viene dada en el compuesto. ¿Cuál es esta relación? La palabra compuesta no lo dice: podría ser un «cesto para papel», un «cesto de papel», un «cesto con papeles», etc. Ya Brugmann observaba que -como es sabido- se tiende a encontrar demasiado significado en las palabras compuestas y, precisamente, mucho más del que encuentran los hablantes de las lenguas en cuestión; esto ocurre, sobre todo, en la traducción de una lengua a otra. Jespersen observaba, por su parte, que los 
compuestos expresan relaciones entre nociones, aunque no precisan el tipo exacto de tales relaciones. Pero ha sido sobre todo el lingüista polaco Norbert Morciniec quien más detenidamente se ha ocupado de este problema, en su libro Die nominalen Wortzusammensetzungen in den westgermanischen Sprachen, Breslau, 1964. Morciniec llega a la conclusión de que, desde un punto de vista estrictamente lingüístico, Sommerhut sólo significa «sombrero determinado por verano», lo que equivale a una confirmación de la relación que ya se veía en la lingüística tradicional entre un «determinatum» y un «determinans». Las determinaciones ulteriores que conducen a la interpretación exhaustiva de los compuestos, respecto a su función de designación en oraciones concretas, vienen dadas -según Morciniec- por el conocimiento general y específico de las cosas; así, por ejemplo, en el caso de Goldwaage, sabemos por el conocimiento general de las cosas que se tratará muy probablemente de una balanza para oro o de oro y, en determinada situación, podremos constatar que se trata precisamente de una balanza de oro. A esto añadiríamos aún, entre el nivel del significado dado por el sistema lingüístico y el del conocimiento general de las cosas, la eventual fijación por la norma de la lengua (cf. nuestro artículo «Bedeutung und Bezeichnung im Lichte der strukturellen Semantik», en P. Hartmann y H. Vernay, Sprachwissenschaft und Übersetzen, Munich, 1970, págs. 111, 116-117)³. Así, las palabras Hauptstadt y Hauptmann, que en sí mismas sólo significan «ciudad principal»y «hombre principal», son interpretadas como «capital» y «capitán» únicamente en virtud de la norma tradicional; fr. pommier es «un $x$ que produce manzanas» (y no, por ejemplo, «un $x$ que vende manzanas»), gracias a la norma de la lengua, y es un «árbol que produce manzanas» en virtud del conocimiento de las cosas. El hecho de que, por ejemplo, Papierkorb haya sido muy verosímilmente creada precisamente con y para la designación «cesto para papel» (las palabras compuestas son creadas, en efecto, en razón de las necesidades de la designación) no importa en lo relativo a su significado, puesto que la lengua alemana, por medio del procedimiento compositivo en cuestión, no expresa esa relación concreta, sino únicamente una relación mucho más general, de tal modo que, una vez creada la palabra Papierkorb, puede ser empleada también para otras designaciones, aparte de la que ha determinado su creación. Creamos por necesidades del habla, pero creamos en la lengua.

2.3.3. Concierne igualmente a la designación la distinción establecida por Charles Bally entre «transposición funcional» (gramatical o sintáctica) y «transposición semántica» (Linguistique générale et linguistique française, pág. 116), en casos como chaleur tropicale, végétation tropicale, según se trate de la vegetación y del calor propios de la zona de los trópicos o bien de una vegetación y de un calor análogos a los que encontramos en estas regiones. Creemos que, en realidad, esta distinción está fuera de lugar y que en este caso no hay sino un mismo valor lexemático, aunque es un valor ampliado respecto a la base de «derivación» (des tropiques). Se trata del fenómeno de la «desconcentración» del significado, fenómeno característico del «desarrollo» (cf. 3.2.1.), en virtud del cual, el campo de designación de un desarrollo es más amplio que el de su base. Así, por ejemplo, it. d'inverno significa «perteneciente al invierno» (cf. giornata d'inverno), mientras que el término

\footnotetext{
${ }^{3}$ N. de la T.: En traducción española: Principios de semántica estructural. Madrid: Gredos, 1977, págs. 195-196, 203-204.
}

Odisea, $n^{\circ} 3,2003$ 
desarrollado invernale significa tanto «perteneciente al invierno» como «parecido a lo que pertenece al invierno» (cf. giornata invernale).

2.4. El estudio de la formación de palabras realizado simultáneamente desde el punto de vista de la expresión y desde el punto de vista del contenido es -como hemos vistonecesariamente incoherente. Al contrario, es posible, sin duda, un estudio coherente realizado exclusivamente desde el punto de vista de la expresión material. Sin embargo, tal estudio no tendría ningún interés desde el punto de vista funcional -de ahí que no lo tuviera tampoco para la comprensión y la descripción fundada y adecuada de las lenguas-, porque entre los tipos de procedimientos materiales (prefijación, sufijación, infijación, adición) y los tipos funcionales o semánticos, no hay ningún paralelismo necesario: distintos procedimientos materiales pueden corresponder a un mismo tipo funcional y a la inversa. Además, un estudio estrictamente material de la formación de palabras -es decir, un estudio que excluyera efectivamente toda referencia al contenido- atribuiría, por una parte, demasiado y, por otra parte, demasiado poco al fenómeno en cuestión. Tal estudio, por un lado, debería concernir también a ciertas yuxtaposiciones y coalescencias casuales (tales como [un] sauve-qui-peut, rendez-vous, bonjour, manu tenere $\rightarrow$ mantenere, pedis ungula $\rightarrow$ esp. pezuña, maris lucius $\rightarrow$ it. merluzzo, it. mezzaluna, esp. correveidile) que, sin embargo, no constituyen procedimientos formativos en el sentido propio del término. Por otro lado, ese estudio no podría identificar como procedimientos formativos materiales algunos tipos que, no obstante, sí lo son, tales como las derivaciones regresivas del tipo fr. garde, esp. guarda $\mathrm{y}$, en general, las formaciones con derivativo cero. Por consiguiente, tal estudio no podría responder adecuadamente al fenómeno de la formación de palabras.

3.1. Al contrario, un estudio realizado desde el punto de vista del contenido puede ser perfectamente coherente y, al mismo tiempo, puede responder exactamente a este fenómeno, ya que la identificación de los procedimientos materiales depende de la identificación de los procedimientos semánticos. Además, sólo un estudio de este tipo puede explicar la naturaleza propia y específica del campo de la formación de palabras.

Desde este punto de vista, la formación de palabras constituye, en el interior del léxico, el campo de las relaciones paradigmáticas secundarias (respecto a las relaciones paradigmáticas primarias, que son las de campo léxico y clase léxica) y corresponde a una gramaticalización del léxico «primario», es decir, de las unidades que constituyen el punto de partida o la base de cada procedimiento formativo (pero que, por supuesto, pueden ser a su vez unidades ya «gramaticalizadas» por un proceso formativo anterior): en todo tipo de formación de palabras, ciertas unidades del léxico son sometidas a una determinación de naturaleza gramatical y con esta determinación implícita son, por así decirlo, «devueltas» de nuevo al léxico; «devueltas» en el sentido de que pueden recibir, a su vez, determinaciones gramaticales explícitas propias de las unidades «primarias». Así, por ejemplo, maisonnette implica una determinación «gramatical» de maison, pero, al mismo tiempo, es un término que, en principio, puede asumir las diferentes determinaciones gramaticales explícitas del término maison.

Las relaciones gramaticales -0 , mejor dicho, «paragramaticales»-, dentro de los productos de la formación de palabras, resultan de las equivalencias semánticas existentes

Odisea, $\mathrm{n}^{\circ} 3,2003$ 
entre estos productos y las construcciones (paráfrasis) explícitas correspondientes, como, por ejemplo, en el caso de beauté: «le fait d'être beau / belle». A este respecto, hay que señalar, no obstante, que las fórmulas de este tipo tienen una función analítica o metalingüística; así «fait», en la fórmula que acabamos de proponer, no es la palabra fait del lenguaje primario, que sería posteriormente determinada por «être beau»; es únicamente el nombre de la sustantivación que implica el desarrollo beau / belle $\rightarrow$ beauté. Del mismo modo, «être» no es sino el nombre de la predicación atributiva y «beau / belle» es el nombre de la unidad beau $(x)+$ belle(s) del lenguaje primario, es decir, el nombre de un beau sin género ni número. En un sentido más abstracto, nuestra fórmula, por consiguiente, significa aproximadamente: «beau $(x)$ - belle $(s)$, gramaticalizado por una predicación atributiva $\mathrm{y}$, después, sustantivado». Por su parte, las relaciones existentes entre las bases de los procesos formativos y sus productos resultan de la oposición semántica entre estas bases y las equivalencias de los productos respectivos; así, beauté, respecto a beau $(x)$ - belle(s), contiene además los rasgos de «predicatividad» y «sustantividad». Por lo tanto, los productos de la formación de palabras no son nunca semánticamente equivalentes a su base y, por ello, no pueden ser obtenidos - como se cree a veces- por medio de «transformaciones que no cambian el significado»: los productos de los procesos formativos tienen siempre un contenido más rico que el de su base léxica.

3.2.1. Los tipos de procedimientos formativos semánticos corresponden a la naturaleza y a las condiciones de la «gramaticalización» que implican. En efecto, por medio de dos criterios que se entrecruzan, podemos distinguir tres tipos fundamentales de formación de palabras, según que la gramaticalización implícita concierna a una sola unidad o a dos unidades en la base (modificación o desarrollo / composición) y según que la gramaticalización corresponda a una función «inactual», es decir, no análoga a las funciones proposicionales, o a una función «actual», del tipo proposicional (modificación / desarrollo). De modo más explícito, nuestros dos criterios son: 1) el número de las unidades de base que implica el proceso formativo (una sola unidad o dos unidades); 2) el tipo general de la función gramatical que implica el producto (función «inactual» o «actual»). En el caso de la modificación y del desarrollo, se trata de la gramaticalización de una sola unidad de base; en la composición, tenemos en la base dos unidades ligadas por una relación gramatical.

En la modificación, la función gramatical implícita es una función «inactual» (del tipo del género o del número), mientras que, en el desarrollo, la función implícita es «actual» (del tipo de las funciones «sujeto», «predicado», «complemento»); en la composición, la función gramatical que liga los dos términos puede ser tanto «inactual» como «actual» (cf., por ejemplo, alem. Rotwein, frente a Leser).

3.2.2. Así, por ejemplo, son modificaciones: maison $\rightarrow$ maisonnette, cavallo $\rightarrow$ cavallino, rouge $\rightarrow$ reougeâtre, viridis $\rightarrow$ subviridis, crier $\rightarrow$ criailler, besar $\rightarrow$ besuquear, árbol $\rightarrow$ arboleda, venir $\rightarrow$ revenir, voir $\rightarrow$ prévoir, fallen $\rightarrow$ hinfallen. En la modificación, la categoría verbal de los productos es siempre la misma que la de las bases modificadas (los sustantivos siguen siendo sustantivos, los adjetivos adjetivos, etc.). Y ello es así, precisamente, porque la función gramatical que implica este proceso es «inactual»; es decir, porque 
se trata de una función que concierne a los lexemas de base como tales y no como miembros de proposiciones o de sintagmas. En cambio, son desarrollos: beau (pred.) $\rightarrow$ beauté, partir (pred.) $\rightarrow$ départ, (en) barque $\rightarrow$ embarquer, (d') inverno $\rightarrow$ invernale, (hors) budget $\rightarrow$ extrabudgétaire, (en) riche $\rightarrow$ enrichir $\rightarrow$ enrichissement. En el desarrollo, que parte de lexemas tomados en cuanto miembros de proposiciones o de sintagmas, los productos pertenecen siempre a una categoría verbal distinta a la de la base; tenemos, por ejemplo, sustantivo $\rightarrow$ adjetivo, sustantivo $\rightarrow$ verbo, adjetivo $\rightarrow$ sustantivo, etc. La composición, por su parte, puede ser de dos tipos: prolexemática o lexemática. Es prolexemática si uno de los dos elementos de la base es un prolexema, es decir, una unidad de naturaleza pronominal, y es lexemática, si los dos elementos de la base son lexemas. Así, por ejemplo, son composiciones prolexemáticas: «pronombre sustantivo genérico» (algo así como: «alguien»o «algo») + calculer $\rightarrow$ calculateur, «pronombre» + wecken $\rightarrow$ Wecker, «pronombre» + pomme $\rightarrow$ pommier, mientras que Wein + rot $\rightarrow$ Rotwein, Kopf + dick $\rightarrow$ Dickkopf, Baum + Apfel $\rightarrow$ Apfelbaum son composiciones lexemáticas. La categoría verbal de los compuestos es siempre la misma que la de los elementos «determinados».

3.2.3. Entre estos procedimientos, el más interesante es, sin duda, el del desarrollo. Por una parte, un producto desarrollado puede constituir a su vez el punto de partida de un nuevo desarrollo, de tal modo que, en las lenguas, tenemos normalmente desarrollos en series orientadas, como (en) riche $\rightarrow$ enrichir $\rightarrow$ enrichissement, (en) barque $\rightarrow$ embarquer $\rightarrow$ embarquement, (de la) nation $\rightarrow$ national $\rightarrow$ nationaliser $\rightarrow$ nationalisation. El orden de las etapas del desarrollo en serie es perfectamente identificable en los casos en que hay paralelismo entre la expresión y el contenido, pero hemos de suponer también el mismo orden en los casos en que la expresión no nos ayuda (por ejemplo, en el caso de vivre, vie, vif). Es cierto, sin embargo, que varios desarrollos paralelos pueden corresponder a una misma «etapa». Por otra parte, el conocimiento de los desarrollos en serie, en cuanto procedimientos de lengua, nos permite saltarnos algunas etapas, es decir, nos permite crear términos sucesivos sin que el término implicado anterior exista efectivamente en la norma de la lengua. Así, conociendo series rum. del tipo domn $\rightarrow$ a domni $\rightarrow$ domnie («señor», «ser señor», «cualidad de señor»), podemos formar palabras tales como fetie, «cualidad de doncella» y profesorie, «cualidad de profesor», sin preocuparnos de la existencia o inexistencia de los verbos a feti y a profesori. Del mismo modo, lat. barbatus implica, desde el punto de vista del sistema de la lengua, un verbo *barbare («dotar de barba»), verbo que, al parecer, nunca fue creado. Fr. gasconnade, esp. gauchada e it. americanata implican verbos como *gasconner («actuar como un gascón»), *gauchar o *americanare, verbos que no existen en la norma de esas lenguas. En estos casos, no se trata de sustantivos formados sobre otros sustantivos (Gascon, gaucho, americano): el cambio obligatorio de la categoría verbal viene, en realidad, ya dado, pero con relación a verbos virtuales. Por lo demás, lo que permite encontrar «lagunas» en las series desarrolladas, desde el punto de vista del sistema lingüístico efectivamente realizado, es la orientación definida y de sentido único del desarrollo; lagunas como, por ejemplo, la inexistencia de los adverbios correspondientes a jeune, vieux, âgé, possible, esp. joven, viejo, ancho, lleno, etc. Además, dado que el desarrollo parte siempre de un empleo (al menos, virtual) del elemento base, podemos tener desarrollos diferentes de un mismo elemento, según sus diferentes acepciones o según los dife- 
rentes significados de las formas homófonas (cf., por ejemplo, it. fegato $\rightarrow$ fegatoso, epatico; terra $\rightarrow$ terrestre, terroso, terreno; esp. esperar $\rightarrow$ espera, esperanza); podemos tener también desarrollos que sólo correspondan a ciertas secciones del significado del elemento base (como en el caso de fr. large $\rightarrow$ largement, plein $\rightarrow$ pleinement, donde los adverbios no corresponden a toda la extensión de los adjetivos). Y podemos encontrar, a la inversa, desarrollos homófonos correspondientes a bases diferentes, como en el caso de fr. mortel, «que puede morir» (desarrollo de mourir) y «que puede hacer morir» (desarrollo de tuer). Finalmente, hay que subrayar el hecho de que la base primaria de una serie desarrollada puede ser un elemento perteneciente a cualquier categoría verbal: un sustantivo, un adjetivo, un verbo e incluso un adverbio (como en el caso de fr. vite $\rightarrow$ vitesse); pero hay que subrayar también el hecho de que los puntos de partida no son necesariamente los mismos en todas las lenguas; así, en italiano, tenemos vero $\rightarrow$ verità, mientras que en español tenemos verdad $\rightarrow$ verdadero, lo cual no deja de tener consecuencias en cuanto al empleo de estas palabras (en efecto, en español, «c'est vrai», «il est vrai» se dice es verdad y no es verdadero).

3.3. Se ha señalado ya hace mucho tiempo que en la formación de palabras (o, al menos, en ciertos procedimientos de formación) tiene lugar una «gramaticalización» del léxico. Ya Ch. Wolff (Philosophia prima sive ontologia, Francfort y Leipzig, 1730, II, 3,3, § 967) considera los «signa derivativa» como «definitionum ac propositionum vicaria». Wolff atribuye esto al hecho de que los «signa derivativa» son motivados de modo extralingüístico, es decir, por algunas propiedades y relaciones de las cosas designadas («Quoniam signa derivativa... significatum primitivum ab arbitrio significatum imponentis, derivativum autem a rebus significatis habent... ideo respectu illius artificialia sunt..., respectu hujus naturalia imitantur»), lo cual constituye una idea aún más antigua (se encuentra ya en F. d'Oliveira, Grammatica da lingoagem portuguesa, Lisboa, 1536, cap. 39), que procede, muy probablemente, de la Antigüedad (verosímilmente de Varrón). También es sabido que Georg von der Gabelentz (Die Sprachwissenschaft, Leipzig, 1901, págs. 463-470) hablaba de la composición nominal como de una «Verwandlung der Sätze in Satztheile». Asimismo, sabemos que Brugmann y Jacobi admitían relaciones sintácticas implícitas en las palabras compuestas, y que Couturat, Jespersen, Porzig, Bally, Kuryłowicz y otros estudiosos identificaron determinaciones gramaticales implícitas; en particular, en las palabras «abstractas», es decir, en los desarrollos predicativos del tipo arrivée, beauté, etc. Pero hay que señalar dos cosas al respecto. En primer lugar, que la gramaticalización no se constata solamente en ciertos procedimientos formativos, sino en todo el campo de la formación de palabras: la gramaticalización es, en efecto, lo que caracteriza a la formación de palabras y lo que la convierte en un campo particular dentro del léxico; e incluso los tipos de procedimientos formativos corresponden a las modalidades de gramaticalización que implican. En segundo lugar, hemos de señalar que, aunque en la formación de palabras hay sin duda una "gramática del léxico», esta gramática no debe confundirse con la gramática en sentido estricto, ya que, en este caso, se trata, realmente, de otras funciones «gramaticales» y no de las que se presentan en la morfosintaxis de una lengua.

Por tal razón, preferimos no hablar de funciones «gramaticales», sino de funciones «paragramaticales». Así, por ejemplo, la formación colectiva implica, sin duda, una «plurali-

Odisea, $n^{\circ} 3,2003$ 
zación», pero no se trata en tal caso de un simple «plural», sino más bien de una «pluralidad que existe como unidad y que es considerada como tal» (arboleda no es simplemente árboles ni Schrifttum equivale a Schriften). Del mismo modo, Rotwein no es exactamente lo mismo que roter Wein (Rotwein es un tipo de vino, mientras que un roter Wein podría ser también un vino con colorante artificial). Las formaciones del tipo départ, Abfahrt implican ciertamente un verbo en función predicativa, pero no implican modo ni tiempo ni número ni persona. Por este motivo, tales formaciones no pueden de ningún modo derivar de frases concretas como Jean part, Hans fährt ab, Jean est beau, Marie est belle, etc., sino únicamente de una función predicativa genérica de partir, abfahren, beau(x) / belle(s). Igualmente, Papierkorb implica una «función preposicional», pero no implica ninguna preposición determinada; calculateur y Wecker contienen un elemento pronominal genérico («quelqu'un ou quelque chose», «jemand oder etwas»), pero éste no se realiza como tal en francés y en alemán. Por tal razón, precisamente, existe la formación de palabras: porque se trata de expresar funciones más genéricas o funciones diferentes de las que son expresadas en la gramática de la respectiva lengua.

3.4.1. Según las funciones «gramaticales» más específicas que impliquen, los tres (o cuatro) tipos fundamentales de formación de palabras pueden ser divididos en subtipos. Así, en la modificación puede tratarse de un cambio de clase o de género (por ejemplo, «verbo transitivo» $\rightarrow$ «verbo intransitivo», König $\rightarrow$ Königin) o bien de una cuantificación; y la cuantificación puede ser, a su vez, formación diminutiva (maison $\rightarrow$ maisonnette, maigre $\rightarrow$ maigrichon, pleurer $\rightarrow$ pleurnicher, con la variante de la apreciación aproximativa: vieux $\rightarrow$ vieillot, grande $\rightarrow$ grandotto), formación aumentativa (libro $\rightarrow$ librone, vecchio $\rightarrow$ stravecchio), formación colectiva (quercia $\rightarrow$ querceto, scatola $\rightarrow$ scatolame), formación intensiva (jour $\rightarrow$ journée), repetición (voir $\rightarrow$ revoir), negación (utile $\rightarrow$ inutile, content $\rightarrow$ mécontent, faire $\rightarrow$ défaire), parcialización (voir $\rightarrow$ prévoir, fallen $\rightarrow$ hinfallen), etc. En el caso del desarrollo, podemos distinguir, según la función sintáctica implícita de la base, el desarrollo predicativo (partir $\rightarrow$ départ, beau $\rightarrow$ beauté), el desarrollo atributivo (des tropiques $\rightarrow$ tropical, d'inverno $\rightarrow$ invernale), el desarrollo de objetos preposicionales (en barque $\rightarrow$ embarquer, de [la] barque $\rightarrow$ débarquer, en riche $\rightarrow$ enrichir, auf [den] Tisch $\rightarrow$ auftischen), etc. Y en la composición, podemos distinguir, por ejemplo, la composición de concordancia y la composición de rección, con varios subtipos.

En el caso del desarrollo, en particular, otras subdivisiones proceden de ciertas determinaciones propiamente gramaticales de la base, que pueden conservarse en los productos resultantes de los procesos formativos. Así, por ejemplo, lat. victoria implica la voz activa de la base vincere, mientras que lat. clades implica la voz pasiva (cf., también, fr. vaincre $\rightarrow$ victoire, it. vincere $\rightarrow$ vittoria, esp. vencer $\rightarrow$ victoria, frente a défaire $\rightarrow$ défaite, sconfiggere $\rightarrow$ sconfitta, derrotar $\rightarrow$ derrota). Del mismo modo, se puede comprobar la conservación de la reflexividad (s'obstiner $\rightarrow$ obstination), del aspecto resultativo (blesser $\rightarrow$ blessure, it. ferire $\rightarrow$ ferita), de la frecuentatividad (piller $\rightarrow$ pillard), de la eventualidad pasiva (manger $\rightarrow$ mangeable), de la semelfactividad (esp. martillar $\rightarrow$ martillazo, it. coltello $\rightarrow$ [verbo] $\rightarrow$ coltellata), etc. A todo ello hay que añadir, además, el procedimiento de destacar («topicalización») tal o cual relación sintáctica de la base (por ejemplo, destacar el objeto: it. pesce $\rightarrow$ pescare; el lugar: fr. loger $\rightarrow$ logement; el tiempo: fr.faucher $\rightarrow$ fauchaison, fleurir 
$\rightarrow$ floraison), la limitación total o parcial a determinados campos de designación (por ejemplo, agricultura, operaciones técnicas, política, administración) y, finalmente, las eventuales «lexicalizaciones» (fijaciones) particulares; cf. al respecto, la tesis doctoral de nuestro discípulo Jens Lüdtke, Prädikative Nominalisierungen mit Suffixem im Französischen, Katalanischen und Spanischen, Tubinga, 1978. Y es interesante observar que estas determinaciones complementarias, en el caso del desarrollo predicativo (así como en otros casos más), se presentan precisamente en el orden que acabamos de señalar (aunque, naturalmente, algunas de ellas e, incluso, todas las determinaciones -salvo la función predicativa misma- pueden faltar en algún desarrollo particular).

3.4.2. Otra propiedad de los procedimientos de formación de palabras es la de poder combinarse entre ellos, incluso varias veces; en este caso, el significado del producto final depende del orden de las combinaciones (incluidas las combinaciones que no existen como palabras autónomas en la norma de la lengua). Así, por ejemplo, en alem. Durchgang tenemos: gehen $\rightarrow$ durchgehen (modificación) $\rightarrow$ Durchgang (desarrollo); en it. passeggiatina: passeggiare $\rightarrow$ passeggiata (desarrollo) $\rightarrow$ passeggiatina (modificación). Cf. también alem. los + Rat $\rightarrow$ ratlos (composición) $\rightarrow$ Ratlosigkeit (desarrollo); Garten + Kinder $\rightarrow$ Kindergarten (composición lexemática) $\rightarrow$ Kindergärtner (composición prolexemática) $\rightarrow$ Kindergärtnerin (modificación); Schule + Volk $\rightarrow$ Volksschule (composición lexemática) + Lehrer (compuesto prolexemático) $\rightarrow$ Volksschullehrer (composición lexemática). A este fenómeno corresponde también el tipo coupe-papier, abundante en las lenguas románicas: en efecto, este tipo combina un compuesto prolexemático coupe- (que corresponde, más o menos, a «coupeur») con otro lexema, en un compuesto lexemático. Lo que caracteriza a este tipo es el hecho de que, en la fase de composición lexemática, se suprimen el derivativo explícito del compuesto prolexemático, así como, muy frecuentemente, las preposiciones que aparecerían en las construcciones equivalentes en sintaxis libre (por ejemplo: coupeur de papier). Es decir que, desde el punto de vista del contenido, este tipo de compuestos es exactamente análogo a los tipos alem. Federhalter e it. pescivendolo, que consisten también en un compuesto prolexemático (Halter, -vendolo) en composición lexemática con otro lexema; cf. nuestra contribución ${ }^{4}$ «Inhaltliche Wortbildungslehre (am Beispiel des Typs coupe-papier)», en Perspektiven der Wortbildungsforschung, publ. por H. E. Breckle y D. Kastovsky, Bonn, 1977, págs. 48-61.

3.4.3. Hay que señalar, además, que formaciones equivalentes en la designación pueden corresponder, en lenguas diferentes ( $\mathrm{y}$, en parte, también en una misma lengua), a procedimientos formativos o combinaciones de procedimientos no equivalentes y, por consiguiente, a significados bastante diferentes. Así, por ejemplo, fr. sans-gêne (adj.) es un desarrollo de sans gêne, mientras que alem. schamlos es un compuesto; fr. débarquer es un desarrollo primario de de [la] barque, mientras que esp. desembarcar es una combinación de un desarrollo (en barco $\rightarrow$ embarcar) con una modificación $(\rightarrow$ desembarcar $)$. Menos

\footnotetext{
${ }^{4}$ N. de la T.: En su traducción al español: «La formación de palabras desde el punto de vista del contenido (A propósito de tipo «coupepepen»)», Gramática, semántica, universales. Estudios de lingüistica funcional, Madrid: Gredos, 1978 [2 ${ }^{\mathrm{a}}$ ed.: Madrid: Gredos, 1987, págs. 239-264].
}

Odisea, $n^{\circ} 3,2003$ 
profunda de lo que se piensa es, sin embargo, la diferencia existente entre pommier y Apfelbaum, arrosoir y Gießkanne; no se trata, en estos casos, de «derivación» y «composición», sino de dos tipos de formación muy próximos; precisamente, de los dos tipos fundamentales de composición: pommier y arrosoir son composiciones prolexemáticas, mientras que Apfelbaum y Gießkanne son compuestos lexemáticos. Equivalencias de este tipo pueden constatarse también en el interior de una misma lengua (cf. alem. Händler y Handelsmann).

4. Lo que acabamos de exponer y, en particular, lo que hemos señalado en el apartado 3.4.1 significa que la formación de palabras es un campo autónomo de la lengua, que incluye aspectos «paragramaticales» y aspectos puramente léxicos; que se trata de un continuo jerárquicamente ordenado, que comienza por los tipos fundamentales de procedimientos formativos y llega hasta las fijaciones particulares de tal o cual lexema. El estudio de la formación de palabras no puede ser sino una rama autónoma de la semántica funcional, que comienza por las funciones paragramaticales de los procedimientos formativos y llega descendiendo hacia lo particular- hasta las fijaciones en la designación. Por consiguiente, la alternativa en el estudio de la formación de palabras entre el nivel de la sintaxis o el nivel del léxico - que, hoy en día, tanto preocupa a los generativistas de diversos colores- es una alternativa desprovista de sentido y de fundamento racional, y es falsa ya en cuanto alternativa, puesto que la formación de palabras, simplemente, no puede pertenecer «o bien a la sintaxis o bien al léxico». En la llamada «sintaxis»-aun haciendo abstracción del hecho de que las funciones gramaticales no son las mismas en la formación de palabras y en la gramática (en sentido estricto)-, no se pueden comprobar ni justificar, por ejemplo, los campos designativos ni las «lexicalizaciones». En el nivel del llamado «léxico»-en el que podemos, sin duda, comprobar y, eventualmente, justificar estos aspectos-, es absolutamente imposible determinar los paradigmas funcionales, las funciones paragramaticales de los procedimientos formativos, de tal modo que la unidad semántica de cada uno de estos procedimientos se disuelve en una casuística heterogénea. Y hemos de decir lo mismo en cuanto al análisis llamado «pragmático», el cual, en lugar de partir de las funciones de lengua, parte de las realizaciones y de las restricciones particulares: una pragmática coherente acerca de la formación de palabras, incluso en cuanto pragmática, sólo puede hacerse a partir de la semántica de la lengua, descendiendo desde ella, y no a la inversa. 